\title{
Evaluation von Disease-Management-Programmen - am Beispiel des DMP Diabetes der BARMER
}

C. Graf

Qualitätsmanagement

Schlüsselwörter

Disease Management

DMP

Evaluation

Diabetes mellitus

Keywords

disease management

DMP

Oevaluation

Diabetes mellitus

Institut

BARMER GEK Hauptverwaltung, Wuppertal

Bibliografie

DOI $10.1055 / \mathrm{s}-0031-1286093$

Dtsch Med Wochenschr 2011;

136: S64 - () Georg Thieme

Verlag KG Stuttgart · New York .

ISSN 0012-0472

Korrespondenz

Dr. rer. soc. Christian Graf

Abteilungsleiter Versorgungs-

programme

BARMER GEK Hauptverwaltung

Lichtscheider Straße 89

42111 Wuppertal

Tel. 0800/332060-992972

eMail

christian.graf@barmer-gek.de
Die Einführung von Disease-Management-Programmen in Deutschland, verbunden mit finanziellen Anreizen der Krankenkassen, wurde von Beginn an kontrovers diskutiert. Trotz vieler praktischer Erfahrungen seit 2003 und zahlreicher Erhebungen gibt es daher hohe Erwartungen an einen neutralen und belastbaren Wirksamkeitsnachweis. Dieser kann im Rahmen der gesetzlich vorgeschriebenen Evaluation nicht hinreichend erbracht werden, weil das Evaluationsdesign keine Kontrollgruppen vorsieht und der Vorher-Nachher-Vergleich maßgeblich auf Daten basiert, die ausschließlich innerhalb der Disease-Management-Programme (DMP) dokumentiert werden. Eine aussagekräftige randomisierte Kontrollstudie (RCT) ist gleichwohl aus verschiedenen Gründen nicht möglich.

Das Mittel der Wahl könnte in der Auswertung von Routinedaten der Gesetzlichen Krankenkassen (GKV) liegen, die umfassende Informationen über Prozessparameter (z.B. Medikation, augenärztliche Kontrollen) und Outcomes (z.B. Schlaganfälle, Herzinfarkte, Amputationen, Hospitalisierung, Mortalität, Gesamtkosten) sowohl für die am DMP teilnehmenden Versicherten als auch für Patienten mit einer entsprechenden Erkrankung, die nicht am DMP teilnehmen, enthalten. Die besondere methodische Herausforderung liegt hierbei in der Adjustierung zwischen Interventions- und Kontrollgruppe zu der Zeit vor der DMP-Teilnahme.

Die BARMER hat seit 2006 verschiedene Auswertungen dieser Art zum DMP Diabetes mellitus durchgeführt. Eine aktuelle Studie mit Routinedaten der BARMER untersuchte die Unterschiede in der Entwicklung der Arzneimittel- und Krankenhausausgaben, der Mortalität sowie diabetischer Folgeerkrankungen von 2003 bis 2007. Die Adjustierung zwischen Interventionsgruppe und Kontrollgruppe wurde mittels „Propensity Score Matching" anhand von Routinedaten des Jahres 2003 vorgenommen. Einbezogen wurden Versicherte, die seit 2004 durchgängig in ein DMP Diabetes eingeschrieben waren und Patienten mit Diabeteserkrankung, die niemals eingeschrieben waren. Als Patienten mit Diabeteserkrankung wurden Versicherte gewertet, die im Jahr 2002 bereits diabetesspezifische Arzneimittelverordnungen erhielten. Für diese Studienpopulation ( $\mathrm{n}=39764$ nach Matching) zeigten sich folgende Ergebnisse: Über 4 Jahre verlief die Entwicklung der Krankenhaus- und Arzneimittelkosten für die DMP-Gruppe um insgesamt 332 Euro günstiger als für die Kontrollgruppe der nicht teilneh- menden Patienten mit Diabeteserkrankung. Auch die Sterblichkeit und die Entwicklung von Herzinfarkten, Schlaganfällen, Niereninsuffizienz und Amputationen fielen in der DMP-Gruppe signifikant geringer aus [1].

Es ist zu fordern, dass Evaluationsstudien mit Kontrollgruppendesign anhand von Routinedaten der GKV zukünftig zum Standard der gesetzlichen Evaluation der DMP werden. Diese sollten für alle DMP (Diabetes mellitus Typ 1 und Typ 2, koronare Herzerkrankung, Herzinsuffizienz, Asthma, chronisch obstruktive Lungenerkrankung, Brustkrebs) nach verschiedenen Kohorten (z.B. Jahr der Einschreibung) und Subgruppen (z.B. Schwere der Erkrankung) differenziert durchgeführt werden. Vor allem gilt es hierbei, einen einheitlichen Standard zur Adjustierung relevanter Risikomerkmale wie Morbidität der Patienten vor Interventionsbeginn zu definieren.

Autorenerklärung: Der Autor ist Beschäftigter der BARMER GEK. Ein finanzieller Interessenkonflikt besteht nicht.

Literatur

1 Stock S, Drabik A, Büscher G, Graf C, Ullrich W, Gerber A, Lauterbach $K W$, Lüngen $M$. German diabetes management programs improve quality of care and curb costs. Health Aff (Millwood) 2010; 29: 2197 2205 